\title{
POST-STROKE COGNITIVE IMPAIRMENT - PHENOMENOLOGY AND PROGNOSTIC FACTORS
}

\author{
Maya Danovska ${ }^{1}$, Boyko Stamenov ${ }^{1}$, Margarita Alexandrova ${ }^{2}$, Dora Peychinska ${ }^{1}$ \\ 1) Department of Neurology, University Hospital - Pleven, Bulgaria \\ 2) Department of Biophysics, Medical University, Pleven, Bulgaria
}

\begin{abstract}
Stroke patients are at higher risk of developing cognitive impairment. Cognitive dysfunctions, especially progressive ones, worsen stroke prognosis and outcome. A longitudinal follow-up of cognitive disorders, however, is rendered difficult by their heterogeneity and the lack of definitions generally agreed upon. Stroke is a major cause of cognitive deficit. The identification of risk factors, clinical determinants and laboratory markers of post-stroke cognitive deficit may help detect patients at increased risk of cognitive deterioration, and prevent or delay the occurrence of poststroke cognitive impairments. Though inflammatory processes have been implicated in the pathogenesis of stroke, their role in the complex pathophysiological mechanisms of post-stroke cognitive impairment is not completely understood. Evidence suggests that elevated serum Creactive protein is associated with both the increased risk of stroke and post-stroke cognitive deficit. The hypothesis of a possible relationship between markers of systemic inflammation and cognitive dysfunctions raises the question of how rational the option of applying non-steroidal antiinflammatory drugs in a proper therapeutic window will be, especially during the acute phase of stroke, to prevent cognitive decline and dementia.
\end{abstract}

Key words: stroke, cognitive impairment, dementia, inflammation, CRP.

\section{INTRODUCTION}

Stroke is the leading cause of severe physical disability in the elderly, the second most common cause of dementia and the third most common cause of mortality after cardiovascular disease and cancer in industrialized countries. ${ }^{1,2}$ The incidence of cognitive deficit increases threefold after stroke and about $25 \%$ of stroke patients develop dementia. ${ }^{3,4,5}$ Some of the patients recover completely from physical disability after the stroke but are often unable to cope with activities of daily life because of cognitive impairment. Current global trends of population ageing and decline in stroke mortality result in increased incidence of poststroke cognitive impairment, thus significantly increasing health care expenditure. There are still no adequate answers to questions such as why stroke patients are at higher risk of cognitive impairment, and what mechanisms characterize the development of dementia.

The clinical presentation of post-stroke cognitive impairment varies from focal neurological deficit to overall cognitive dysfunction. ${ }^{4,6}$ Testing and assessment of cognitive functions is often quite difficult because of lack of generally accepted definition of cognitive impairment and information about the pre-stroke cognitive state of the patients. A better understanding of the complex relationship between stroke and cognitive deficit might provide additional options for prevention and delay of disabling post-stroke symptoms. Identification of diagnostically reliable risk factors and clinical determinants of cognitive impairment in the acute phase of stroke is a successful approach in early detection of patients at increased risk of cognitive deterioration and a step forward in preventing the progress of the post-stroke cognitive impairments.

\section{POST-STROKE COGNITIVE IMPAIRMENT- DEFINITION, TERMINOLOGY, CLASSIFICATION \\ Attempts to classify post-stroke cognitive} dysfunctions are impeded by serious terminology-related problems because of their heterogeneity, high incidence, social significance and uniqueness. ${ }^{7,8}$ There exist many definitions of post-stroke cognitive disorders, mostly based on concepts of cognitive decline and dementia. Post-stroke cognitive impairment (PSCI) is defined as a cognitive deficit that develops up to the third month after stroke with a minimal duration of six months which is not associated with any other condition or disease, e.g. metabolic and endocrine disorders, vasculitis, depression. ${ }^{9,10}$ Post-stroke cognitive deficits are generally subdivided into cognitive impairment no dementia (CIND), and dementia when performance of daily activities and social functions are impaired. NINDS-AIREN criteria (National Institute of Neurological Disorders and Stroke - Association Internationale pour la Recherchй et l'Enseignement) ${ }^{11}$ define dementia as a cognitive decline from a previously higher level of memory functioning and two or more other cognitive domains; the decline being severe enough to interfere with normal activities of daily life. ${ }^{12}$ The 
term Post-Stroke Dementia (PSD) is used to denote all types of dementia following stroke, no matter whether the etiology is vascular $(\mathrm{VaD})$, degenerative $(\mathrm{AD})$ or mixed dementia (Mixed D). ${ }^{4}, 13,14,15$ The dementia following ischemic stroke or brain hemorrhage, irrespective of their location is called Vascular Dementia (VD). ${ }^{16,17}$ Vascular Dementia as a concept does not cover all grades of cognitive deficit after stroke, thus imposing certain difficulties and restrictions. Consequently, a new more appropriate concept of Vascular Cognitive Impairment (VCI) was suggested, defining every type of cognitive deficit associated with, or caused by cerebrovascular disease or vascular risk factors, regardless of their severity. ${ }^{11,18,19,20}$ Despite the disagreement of some authors, stating that the differences between vascular cognitive impairment and the rest of cognitive disorders are still obscure and should be further clarified, the term of VCI as a concept is gaining much popularity in routine neurology practice. ${ }^{19,21,22}$

\section{EPIDEMIOLOGYAND FREQUENCY}

The risk of developing cognitive deficit increases after stroke, and $25-50 \%$ of the stroke survivors develop poststroke dementia. ${ }^{4,5,23,24}$ According to Erkinjuntti et al. ${ }^{25}$, the frequency of dementia after stroke is 4-6 times higher than the frequency of dementia in patients of the same age but without a history of stroke. A higher frequency of post-stroke cognitive deficit has also been found during the third month after stroke onset as compared to the frequency one year after the stroke. This is probably due to the higher mortality rate in post-stroke patients with cognitive deterioration. ${ }^{4}$ Vascular dementia accounts for $15-25 \%$ of all dementia types, and the incidence is highest in Asia. ${ }^{3}$ The incidence of vascular dementia is approximately 3.8 per 1000 annually, although great variations are possible depending on study methodology and diagnostic criteria. Comparatively higher frequency of vascular dementia has been observed in rural areas, usually related to lower educational level of the population, lower quality of health care service and the effect of isolation. The frequency of vascular dementia doubles every 5.3 years, ${ }^{26}$ and is twice as frequent in patients with a low level of education. ${ }^{27}$ The incidence of post-stroke dementia is $6.7 \%$ in the age range $60-64$ years and $26.5 \%$ in patients over 85 years of age $e^{4,28,29}$ Data concerning genderrelated prevalence of post-stroke cognitive impairments are contradictory. ${ }^{4,13,30}$ Regardless of the great variations in the incidence and epidemiology of post-stroke dementia, data from longitudinal studies confirm the higher risk of developing cognitive impairment after stroke. ${ }^{4}$

\section{OF STROKE}

COGNITIVE DISORDERS IN THE ACUTE PHASE

Accurate assessment of cognition in the acute phase of stroke is very difficult because of fluctuations characterizing the post-stroke cognitive state. ${ }^{31}$ The type and severity of cognitive disorders in the acute phase basically depend on stroke features. Location and size of lesion, and the adequacy of cerebral perfusion determine the risk of cognitive impairment after stroke. Reduction in regional blood flow causes impaired cognitive performance, while restoration of cerebral perfusion improves the cognitive state. ${ }^{32}$ In general, hypoperfusion and acute infarction result either in focal cognitive dysfunctions such as aphasia, neglect and apraxia, or in overall cognitive dysfunctions such as disorders of memory, attention and executive functions and other cognitive domains. ${ }^{33}$ According to some authors, the frequency of cognitive disorders in the acute phase of stroke is over $70 \% .^{34,35}$ Yet a number of acute cognitive dysfunctions are resolved or improved during the first weeks and months after stroke owing to either re-canalization followed by reperfusion, or cerebral plasticity. ${ }^{32}$ It should be emphasized that cognitive impairments presented in the acute phase of stroke are mostly determined by the local effects of the lesion or hypoperfusion. Of course, stroke patients have different baseline cognition and comorbidities, which may play a role regarding the severity of post-stroke deficit. Some demographic and medical factors may also significantly influence the post-stroke cognitive performance in the acute period of stroke. Advanced age appears to be an important predictor of poor cognitive outcome after stroke. ${ }^{35}$ Furthermore, age even predicts the cognitive deterioration and dementia in the chronic phase of stroke. Data concerning the influence of some clinical parameters as hyperglycemia, hyperpyrexia and seizures on the level of cognitive performance are contradictory and scarce. ${ }^{35}$ Sometimes the accurate evaluation of the cognitive state in the acute phase of stroke is further complicated because of the effect of medications such as tranquilizers and sedating agents that worsen cognitive performance. Many therapeutic interventions in acute stroke like intravenous thrombolysis might positively influence the outcome by reducing disability and improving cognitive status after stroke. ${ }^{36}$

\section{COGNITIVE DISORDERS IN THE CHRONIC PHASE OF STROKE}

While many of the post-stroke cognitive disorders are resolved during the sub-acute period, some of the patients undergo progressive cognitive and physical deterioration, though without any new clinically detectable acute cerebrovascular events. ${ }^{37,38}$ Evidence from epidemiological studies undoubtedly confirms that stroke is a strong predictor of cognitive decline and dementia. ${ }^{39}$ The natural evolution from stroke to dementia goes through a mild vascular cognitive impairment. Approximately $8 \%$ of the patients with mild cognitive impairment after stroke develop dementia within a year. ${ }^{38}$ In general, more severe clinical deficit at stroke onset is associated with a higher risk of post-stroke cognitive impairment. Patients with recurrent and multiple infarctions are also at higher risk of developing cognitive impairment. ${ }^{40}$ 
Left hemispheric supratentorial lesions, anterior and posterior cerebral artery territory infarcts, multiple infarctions and the so-called 'strategic infarctions' are more likely to cause dementia after stroke. Cerebral infarctions that cause dementia, regardless of other factors and without any other lesions are defined as 'strategic infarctions'. The clinical manifestation of post-stroke cognitive impairment in the chronic phase is quite heterogeneous and might be dominated by symptoms of vascular dementia, degenerative dementia or mixed dementia. It is very difficult to answer the question to what extent the cognitive deficit is determined by the stroke effect on the vessels. It is also difficult to explain the complex pathophysiological mechanisms of superimposing stroke vascular effects on previously existing neurodegenerative changes of Alzheimer's type and how the Alzheimer pathology acts synergistically with vascular risk factors to cause cognitive decline.

\section{DETERMINANTS OF POSTSTROKE COGNITIVE IMPAIRMENT}

Cognitive impairment following stroke is common but its determinants are still not fully understood..$^{35}$ Clinical determinants of post-stroke cognitive impairment include: stroke characteristics (pathomorphological and neuroradiological); pre-existing and coexisting vascular risk factors; and individual (demographic and clinical) data (Fig.1).

\section{Stroke characteristics}

Exploring the potential mechanisms of post-stroke cognitive impairment, Tatemichi et al. ${ }^{4}$ have emphasized that stroke location, volume and number of lesions are important factors. According to their findings, the location of stroke is a parameter of greater importance for the cognitive status, if compared to the volume of cerebral lesion. Volume becomes a significant determinant of cognitive impairment only in cases when the compensatory capacity of the organism is exceeded. As far as location is concerned, of special importance for the cognitive deficit are left hemisphere and supratentorial infarctions, followed by lesions in the territory of anterior and posterior cerebral arteries. The analysis of Sydney Stroke Study ${ }^{21}$ shows that the volume of the lesion is a significant determinant of post-stroke dementia while prestroke cognitive functions might be significant determinants of post-stroke cognitive decline during the first three to six months of stroke onset. Usually, massive infarction causes dementia but its clinical course is not progressive in most cases. The number of cerebral lesions has been found to be the third most significant determinant. Multiple infarctions often cause progressive, step-like course of cognitive disorders and eventually lead to multi-infarction dementia. ${ }^{41}$ All previous strokes and multiple lesions undoubtedly increase the risk of post-stroke cognitive impairment. ${ }^{4,42}$ It has been found that a more severe clinical deficit at stroke onset is associated with a higher risk of post-stroke dementia. 43,44 Special attention is paid to the "strategic infarcts", i.e. cerebral infarcts leading to dementia when cortical and subcortical areas important for cognition are damaged. The most frequent strategic locations are left angular gyrus, inferomesial temporal and mesial frontal locations, thalamus, genu of the left internal capsule and caudate nuclei.

\section{Vascular risk factors and individual patient data}

According to results from the New York Study ${ }^{14}$, advanced age is the major determinant of post-stroke cognitive impairment. A dynamic follow-up of stroke patients has demonstrated that $15 \%$ of the patients in the $60-69$ year age group, $26 \%$ of the $70-79$-year-olds and $36 \%$ of those over 80 years of age had new-onset dementia. ${ }^{14}$ Significant differences in gender distribution of patients with cognitive impairment have not been established, although some authors have reported a higher incidence of cognitive disorders in women. ${ }^{4,30}$ Low education level has also been announced as an independent predictor of post-stroke dementia. ${ }^{3,27}$ The risk of dementia is significantly higher in patients with a history of cognitive deficit before stroke onset. ${ }^{30,44,45}$

According to the Sydney Stroke Study 21, cerebrovascular risk factors are not independent predictors of post-stroke cognitive impairment. Systemic arterial hypertension, a major risk factor of stroke, has not been identified as an absolute risk factor for post-stroke deficit. ${ }^{45,46,47}$ Indirect evidence has been published that higher levels of systemic blood pressure produce greater risk of post-stroke dementia. ${ }^{48}$ The PROGRESS study results prove that lowering the blood pressure in patients with a previous stroke reduces the risk of cognitive deficit by reducing the risk of a recurrent stroke. ${ }^{48}$ According to other studies, diabetes, cardiac rhythm disorders, and myocardial infarction might also be independent risk factors of poststroke cognitive impairment. ${ }^{3,4}$ The question about the cause-and-effect relationship between hypoxic-ischemic disorders and progressive cognitive decline remains debatable. The influence of hyperlipidemia, hyperhomocysteinemia, alcohol abuse and smoking on poststroke cognitive deficit has not been proved yet. ${ }^{3}$ Results from population-based and hospital-based studies have shown that patients with post-stroke cognitive impairment and dementia have higher mortality rates than patients without dementia regardless of age and co-morbidities. ${ }^{14}$ For example, the 5-year survival rate of patients with post-stroke dementia is hardly $39 \%$, whereas that of age-matched controls is $75 \%$. The higher mortality rate of patients with stroke and cognitive decline is probably due to the ongoing negative additive influence of vascular risk factors.

\section{Neuroradiological determinants}

Modern neuroimaging techniques are an integral part of the actual diagnostic criteria of cognitive impairment. They help obtain extremely valuable information about the pathomorphological changes associated with post-stroke cognitive decline and dementia. Some authors suppose that "silent" infarctions could predict post-stroke dementia, 
especially in cases when the period between the stroke and the clinical manifestation of cognitive impairment is too long. 4,49 General cerebral atrophy is also associated with increased risk of developing cognitive impairment and more frequently found in patients with pre-stroke dementia (Fig.1) ${ }^{3,4,50}$. The presence and severity of white matter changes have been reported as independent predictors of post-stroke dementia in patients with lacunar and multiple infarctions (Fig.1) ${ }^{4,51}$. Hence, silent infarctions, general cerebral atrophy and white matter changes could be considered as possible predictors of post-stroke cognitive impairment.

To the best of our knowledge, there is still not enough evidence to identify reliable neuroimaging markers for early prediction of post-stroke cognitive deficit. The exact causal relationship between structural abnormalities and cognitive impairment after stroke needs further clarification.

POTENTIAL ROLE OF INFLAMMATION IN PREAND POST-STROKE COGNITIVE DEFICIT

There is growing evidence supporting the hypothesis that stroke triggers complex mechanisms inducing molecular and tissue dysfunctions, which, in combination with concomitant risk factors modified by the demographic characteristics and individual data of the patient, might cause permanent cognitive deficit. ${ }^{52}$ Inflammation is one of the basic mechanisms in the pathogenesis of atherosclerosis and its complications. ${ }^{53}$ On the other hand, cerebral atherosclerotic changes, stroke and leukoaraiosis in particular, may interrupt the integrity of frontal sub-cortical circuits and cause cognitive impairment and depression. ${ }^{53,54,55}$ That is why, inflammation is supposed to be an important contributing factor in both neuronal damage after stroke and the development of amyloid plaques associated with cognitive deficit and dementia in the elderly. ${ }^{53,56,57}$

Defining dementias in the elderly as neurodegenerative (Alzheimer's dementia) or vascular, respectively vascular cognitive impairment (VCI) is very difficult when assessing individual patients. In most cases, there is a simultaneous expression or overlapping of these states, depending on concurrent risk factors and pathophysiological mechanisms. ${ }^{58,59}$ These observations have served as a basis for prospective epidemiological and clinical studies aiming to define the potential relationship between inflammation and cognitive impairment, and to identify systemic and brain inflammatory markers associated with post-stroke cognitive dysfunctions.

Stroke initiates acute local inflammatory response. ${ }^{60}$ Inflammatory molecules such as cytokines, chemokines and complement factors have been found in the CSF of patients with stroke. ${ }^{53}$ It has not been clarified yet as to what extent the post-stroke inflammatory state interacts or potentiates neurodegenerative processes associated with cognitive decline in the elderly. ${ }^{61}$ Though a non-specific inflammatory marker, C-reactive protein (CRP) has been proved to be a predictor of stroke outcome and prognosis (Fig. 2). ${ }^{52,54}$ It is supposed that both acute and chronic inflammation is equally significant for the development of post-stroke dementia and depression. ${ }^{53}$ It is the high sensitivity CRP (hs-CRP), one of the major markers of chronic inflammation, which has been detected in senile plaques and neurofibrillary tangles in Alzheimer's disease. ${ }^{62}$ Growing evidence confirms the hypothesis that CRP is not only an inflammatory marker but also has powerful pro-atherogenic activity and is directly involved in the process of atherogenesis, causing stroke, leukoaraiosis and vascular dementia (Fig.2). ${ }^{63}$ Theoretically, hs-CRP, as a non-specific but highly sensitive marker of the subclinical chronic inflammation, might indicate damage to cerebral microstructures of frontal pathways, associated with cognitive performance and executive functions. ${ }^{64} \mathrm{CRP}$ also promotes the expression of tissue factors in monocytes; opsonizes low-density lipoproteins, impairs endothelial functions, upregulates the expression of endothelial adhesion molecules, thus directly participating in the initiation and acceleration of vascular disease. ${ }^{65,66}$ Experimental studies in vitro have also established that CRP exerts direct neurotoxicity (Fig.2). ${ }^{62}$ The pro-inflammatory response might cause neuronal damage and cerebral atherosclerosis resulting in macro- and micro-angiopathy. Experimental and clinical evidence has confirmed that inflammatory mechanisms play an important role in the pathogenesis and progress of thrombosis, plaque rupture and stroke. ${ }^{54}$ Furthermore, CRP has been correlated to white matter lesions and "silent" infarctions which are responsible for cognitive impairment and dementia ${ }^{67,68}$. A probable causal relationship between the CRP serum level and the post-stroke cognitive decline has been studied prospectively. ${ }^{69,} 70,71,7273$ These studies have found increased CRP level as related to both stroke prognosis and prediction of cognitive decline and dementia. ${ }^{53}$

The possibility of using a laboratory marker such as CRP level in the circulation for early identification of patients at increased risk of post-stroke cognitive impairment seems quite attractive. Up to date, there is no agreement as to the necessity of regular CRP screening of the general population and dynamic follow-up of stroke patients. Epidemiological and clinical studies have confirmed the hypothesis that increased serum CRP level is a risk factor of stroke, cognitive impairment and depression. However, the major causal relationship could be undoubtedly confirmed only after a convincing demonstration that lowering the CRP level in the circulation prevents the development and progress of cognitive dysfunctions.

\section{THERAPEUTIC OPPORTUNITIES AND GUIDELINES FOR FUTURE STUDIES}

Successful drug therapy of post-stroke cognitive disorders remains an unsolved therapeutic challenge, because of the complex pathophysiological mechanisms initiated by the stroke. The treatment, usually continuous and 
multifactorial should aim to restrict stroke expansion and prevent stroke recurrence thus increasing the opportunity of delaying or preventing further cognitive impairment. ${ }^{43}$ Preventive interventions should include efforts to modify risk factors and primary and secondary stroke prophylaxis. Although no convincing evidence exists that modification of vascular risk factors will result in reduction of cognitive impairment, it is appropriate to apply antihypertensive and lipid-lowering medication for the purpose of stroke prophylaxis, thus preventing post-stroke cognitive dysfunctions. Additional specific interventions include systemic control of vasoactive, hemorheologic and metabolic mechanisms implicated in the neuronal damage, related to functional and cognitive stroke outcome. ${ }^{37}$

The hypothesis of a possible relationship between markers of systemic inflammation and cognitive dysfunctions raises the question of how rational the option of applying non-steroidal anti-inflammatory drugs in a proper therapeutic window will be, especially during the acute phase of stroke, to prevent cognitive decline and dementia. ${ }^{74}$ Future prospective studies on the incidence and clinical manifestation of cognitive disorders after stroke might offer possibilities of detecting unknown risk factors of cognitive decline and novel opportunities for successful therapeutic impact. Further investigations on some genetic factors associated with cognitive deterioration and dementia are also necessary. ${ }^{44}$ Of course, some novel therapies for neuroprotection and improvement of the cerebral plasticity need further prospective experimental confirmation and successful clinical translation.

\section{SUMMARY}

Cognitive impairment following stroke is common, but its determinants are not well defined. The major prognostic factors for cognitive decline and dementia are stroke characteristics, pre-existing and co-existing risk factors and individual patients' data. Cognitive impairment in the acute phase of stroke is mostly determined by the location and the size of the lesion. Multiple and recurrent infarctions increase the risk of developing dementia. Advanced age is important predictor of poor cognitive outcome after the stroke. The impact of cerebrovascular risk factors on post-stroke cognitive impairment is debatable and contradictory. No reliable neuroimaging markers for predicting post-stroke deficit are found yet. The potential role of inflammation for pre- and post-stroke cognitive deficit offers new diagnostic and therapeutic approaches for prevention of cognitive decline and dementia. What is of clinical importance now is to design a working algorithm for early assessment of the potential risk of post-stroke cognitive impairment thus laying the foundation for successful prophylaxis and treatment of post-stroke cognitive dysfunctions.

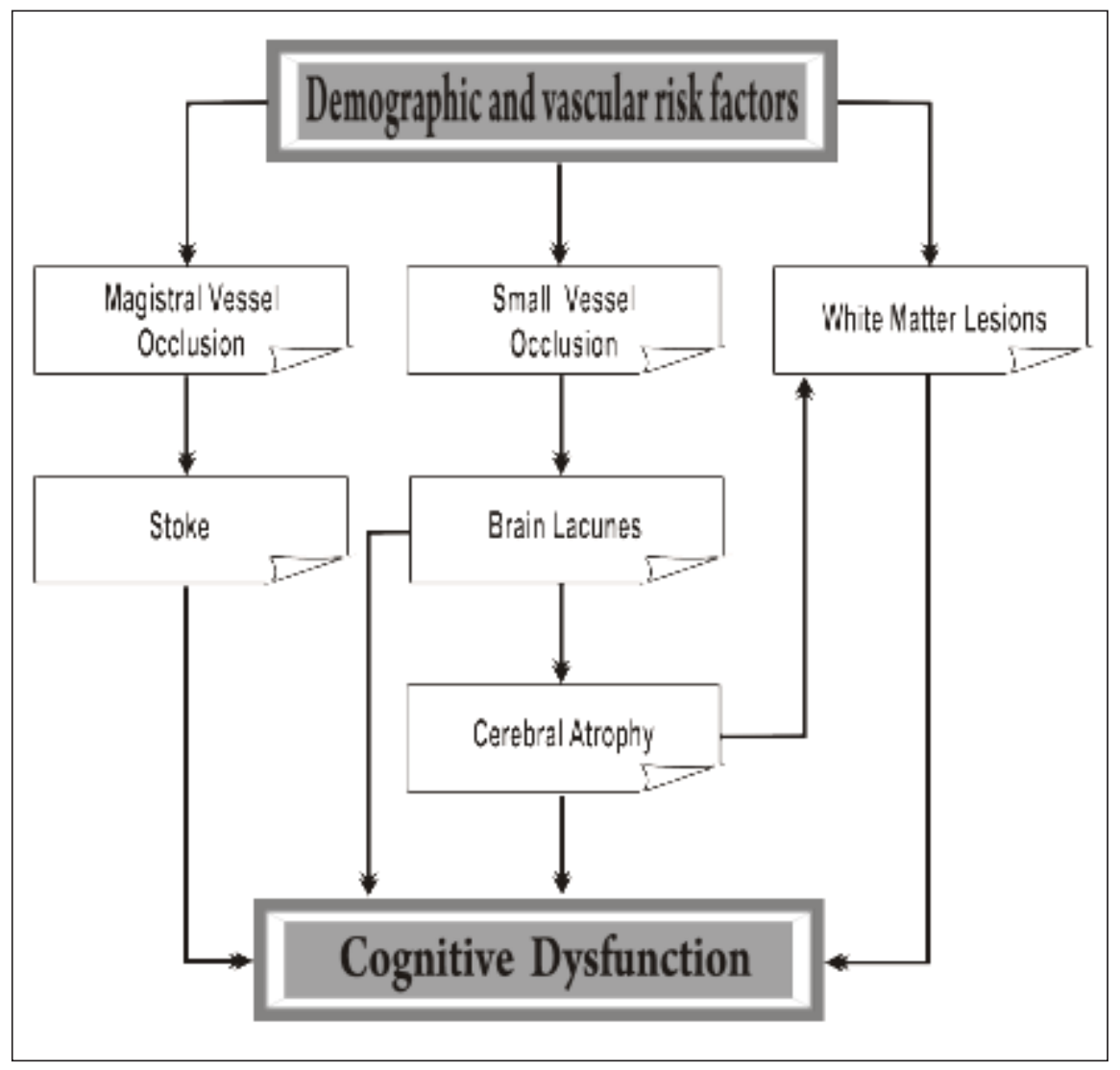

Fig. 1. Hypothetical pathophysiological mechanisms of cognitive dysfunctions.

Major clinical determinants of post-stroke cognitive impairment: stroke features; pre-existing and coexisting vascular risk factors, demographic characteristics. 


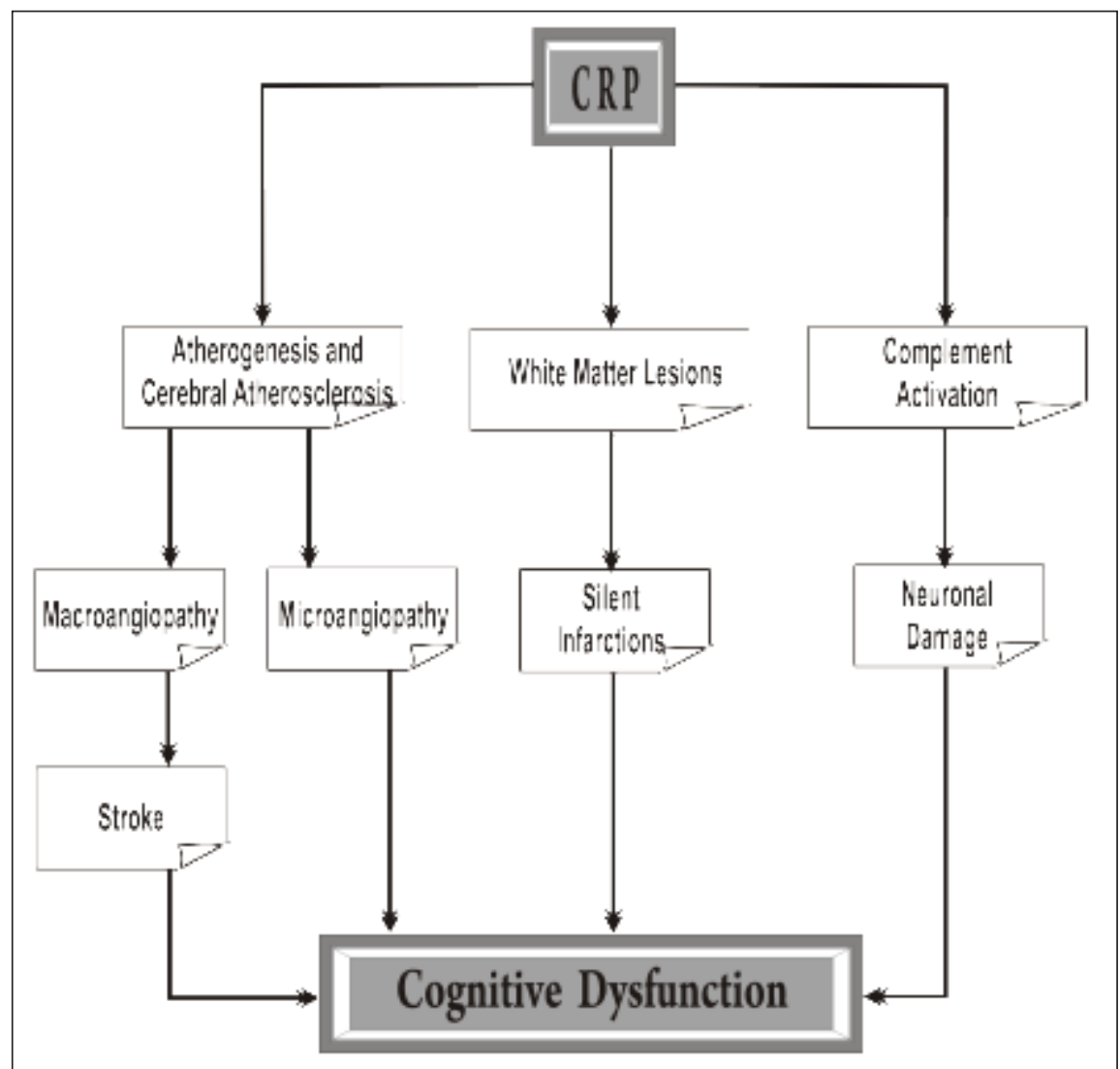

\section{REFERENCES:}

1. Murray CJ, Lopez AD. Global mortality, disability and the contribution of risk factors. Global burden of disease study. Lancet 1997 May 17;349(9063): 1436-42. [PubMed] [CrossRef]

2. Titianova E, Velcheva I, Stamenov B. Treatment of Acute Ischemic Stroke with Thrombolysis in Bulgaria. Neurosonology and Cerebral Hemodynamics. 2010, 6(1):9-14. [in Bulgarian]

3. Desmond DW, Moroney JT, Sano M, Stern Y. Incidence of dementia after ischemic stroke. Stroke 2002 Sep; 33(9):2254-2262. [PubMed] [CrossRef]

4. Tatemichi TK, Desmond DW, Paik M, Figueroa M, Gropen TI, Stern Y, et al. Determinants of dementia related to stroke. Ann Neurol 1993 Jun;33(6):568575. [PubMed]

5. Хаджиев Д. Пьрвична профилактика на исхемичните инсулти и невропротекция. MD 2003. [in Bulgarian]

6. Grant I, Adams KM. Neuropsycho- logical Assessment of Neuropsychiatric and Neuromedical Disorders. Oxford University Press US, 2009; 315-319.

7. Hennerichi M. What are the mechanisms for post-stroke dementia? [PubMed] [CrossRef]

8. Haring HP. Cognitive impairment after stroke. Curr Opin Neurol 2002 Feb,15(1):79-83. [PubMed]

9. Vakhnina NV, Nikitina LY, Parfenov VA, Yakhno NN. Post-stroke cognitive impairment. Neurosci Behav Physiol 2009 Oct;39(8):719-72. [PubMed] [CrossRef]

10. Hachinski V. Vascular dementia: a radical redefinition. Dementia 1994 May-Aug;5(3-4):130-2. [PubMed]

11. Stephan BC, Mathews FE, Khaw KT, Dufuil C, Brayne C. Beyond mild cognitive impairment: vascular cognitive impairment, no dementia(VCIND). Alzheimers Res Ther 2009 Jul 9;1(1):4. [PubMed] Lancet Neurol. 2009 Nov;8(11): 973-975.
Fig. 2. Hypothetical mechanisms associating CRP level, stroke and cognitive dysfunctions.

Inflammation plays a key role in the pathogenesis of atherosclerosis and its complications. Inflammatory processes are involved in stroke, white matter lesions and neuronal damage. $\mathrm{C}$ reactive protein (CRP), a nonspecific marker of systemic inflammation, has been proved to be a predictor of stroke outcome and post-stroke cognitive impairment.
12. Roman GC, Tatemichi TK, Erkinjuntti T, Cummings JL, Masdeu JC, Garcia JH, et al. Vascular dementia: diagnostic criteria for research studies. Report of the NINDS-AIREN International Workshop Neurology 1993 Feb; 43 (2):250-60. [PubMed]

13. Bowler JV. Modern concept of vascular cognitive impairment. $\mathrm{Br}$ Med Bull. 2007; 83:291-305. [PubMed] [CrossRef]

14. Leys D, Henon H, MackowiakCordoliani MA, Pasquier. Poststroke dementia. Lancet Neurol. 2005 Nov; 4(11):752-759. [PubMed] [CrossRef]

15. Pasi M, Poggesi A, Salvadori E, Pantoni L. Post-stroke dementia and cognitive impairment. Front Neurol Neurosci. 2012; 30:65-9. Epub 2012 Feb 14. [PubMed] [CrossRef]

16. Serrano S, Domingo J, RodriguezGarcia E, Castro MD, del Ser T. Frequency of cognitive impairment 
without dementia in patients with stroke. Stroke 2007 Jan;38(1):105-110. [PubMed] [CrossRef]

17. John SM, Gaiane MR, Helmut L. Vascular dementia. Wiley- Blackwell 2001 1-19.

18. Lodder Y. Poststroke cognition and the flight against the hard problem: vascular neurologists, enter the arena! Stroke 2007 Jan;38(1):7-8. [PubMed] [CrossRef]

19. Hachinski V, Iadecola C, Petersen RC, Breteler MM, Nyenhuis DL, Black $\mathrm{SE}$, et al. National Institute of Neurological Disorders and Stroke-Canadian Stroke Network vascular cognitive impairment harmonization standard. Stroke 2006 Sep;37(9):2220-41. Epub 2006 Aug 17. [PubMed] [CrossRef]

20. O’Brien J, Erkinjuntti T, Reisberg B, Roman G, Sawada T, Pantoni L, et al. Vascular cognitive impairment. Lancet Neurol 2003 Feb;2(2):89-98. [PubMed] [CrossRef]

21. Sachdev PS, Brodaty H, Valenzuela MJ, Lorentz L, Looi JC, Berman K, et al. Clinical determinants of stroke and mild cognitive impairment following ischemic stroke: the Sydney Stroke Study. Dement Geriatr Cogn Disord 2006; 21(5-6):275-83.Epub 2006 Feb 10. [PubMed] [CrossRef]

22. Zhao QL, Zhou Y, Wang YL, Dong KH, Wang YJ. A new diagnostic algorithm for vascular cognitive impairment: the proposed criteria and evaluation of its reliability and validity. Chin Med J (Engl) 2010 Feb;123(3):3119.

23. Cumming $\mathrm{T}$, Blomstrand $\mathrm{C}$, Bernhardt J, Linden T. The NIH Stroke Scale can establish cognitive function after stroke. Cerebrovasc Dis 2010; 30 (1): 7-14.

24. Cumming T, Brodtmann A. Dementia and stroke: the present and future epidemic. Intern J of Stroke 2010; 5:453-454.

25. Erkinjuntti T. Vascular cognitive deterioration and stroke. Cerebrovasc Dis 2007; 24 Suppl 1:189-94.

26. Jellinger KA, Attems J. Prevalance and pathology of vascular dementia in the oldest-old. $J$ Alzheimers
Dis 2010. Aug 6.

27. Nunes B, Silva RD, Cruz VT et al. Prevalence and pattern of cognitive impairment in rural and urban populations from Northern Portugal. BMC Neurol 2010 Jun 11; 10-42.

28. Ballard C, Rowan E, Stephens S, Kalaria R, Kenny R. Prospective follow up study between 3 and 15 months after stroke: improvements and decline in cognitive function among dementia-free stroke survivors $>75$ years of age. Stroke 2003; 34: 2440-2445.

29. Snaphaan L., de Leeuw F. Poststroke memory function in nondemented patients: a systemic review on frequency and neuroimaging correlates. Stroke 2007 38:198-203.

30. Tang W, Chan S, Chiu H, Ungvari G, Wong K, Kwok T. Emotional incontinence in Chinese stroke patients: diagnosis, frequency, clinical and radiological correlates. J Neurol 2004; 251:865-69.

31. Gottesman R, Hillis A. Predictors of cognitive dysfunction resulting from ischemic stroke. Lancet Neurol 2010; 9: 895-905.

32. Hillis A, Kleinman J, Newhart M. et al. Restoring cerebral blood flow reveals neural regions critical for naming. J Neurosci 2006; 26: 8069-73.

33. Lee B, Kim E, Ku B et al. Cognitive impairments in patients with hemispatial neglect from acute right hemispheric stroke. Cogn Behav Neurol 2008; 21:73-76.

34. Lesniak M, Bak T, Czepiel W, Seniow J, Czlonkowska A. Frequency and prognostic value of cognitive disorders in stroke patients. Dement Geriatr Cogn Disord 2008; 26:356-63.

35. Nys G, van Zandvoort M, de Kort P, Jansen B, de Haan E, Kappelle L. Cognitive disorders in acute stroke: prevalence and clinical determinants. Cerebrovasc Dis 2007; 23:408-16.

36. Nys G, van Zandvoort M, Algra A, Kappelle, de Haan E. Cognitive and functional outcome after intravenous recombinant tissue plasminogen activator treatment in patients with a first symptomatic brain infarct. $J$ Neurol 2006; 253:237-41.
37. Linden T, Skoog I, Faderberg B, Steen B, Blomstrand C. Cognitive impairment and dementia 20 months after stroke. Neuroepidemiology 2004; 23:45-52.

38. Sachdev P, Chen X, Brodaty H, Thompson C, Altendorf A, Wen W. The determinants and longitudinal course of post-stroke mild cognitive impairment. $J$ Int Neuropsychol Soc 2009;15: 915-23.

39. Troncoso J, Zonderman A, Resnick S, Crain B, Pletnikova O, O'Brien R. Effects of infarcts on dementia in the Baltimore Longitudinal Study of Aging. Ann Neurol 2008; 64:168-76.

40. Pendlebury S, Rothwell P. Prevalence, incidence and factors associated with pre-stroke and poststroke dementia: a systematic review and meta-analysis. Lancet Neurol 2009; 8: 1006-18.

41. Moroney J, Bagiella E, Tatemichi T, Paik M, Stern Y, Desmond D. Dementia after stroke increases the risk of long-term stroke recurrence. Neurology 1997; 48:1317-25.

42. Zhou D, Wang J, Deng J, Chao C, Chen M. Frequency and risk factors of vascular cognitive impairment three months after ischemic stroke in China: the Chogqing Stroke Study. Neuroepidemiology 2005; 24(1-2):87-95.

43. Desmond D, Moroney J, Sano M, Stern Y. Mortality in patients with dementia after ischemic stroke. Neurology 2002; 59: 537-543.

44. Henon H, Durieu I, Guerouaou D, Lebert P, Pasquier P, Leys D. Poststroke dementia: incidence and relationship to pre-stroke cognitive decline. Neurology 2001; 57:1216-22.

45. Duron E, Hanon O. Vascular risk factors, cognitive decline and dementia. Vasc Health Risk Manag 2008; 4:363-381.

46. Potluri R, Natalwala A, Uppal H, Heun R. Different risk factors in vascular dementia and ischemic stroke. Neuroepidemiology 2009; 32:80.

47. Sundar U, Advani S. Post-stroke cognitive impairment at 3 months. Ann Indian Acad Neurol 2010; 13:42-6.

48. Tzourio C, Anderson C, 
Chapman N, et al. Effects of blood pressure lowering with perindopril and indipamide therapy on dementia and cognitive decline in patients with cerebrovascular disease. Arch Intern Med 2003; 163:1069-75.

49. Rasquin S, Verhey F, van Oostenbrugge R, Lousberg R, Lodder J. Demographic and CT scan features related to cognitive impairment in the first year after stroke. J Neurol Neurosurg Psychiatry 2004; 75:1562-67.

50. Ivan C, Sudha S, Beiser A, et al. Dementia after stroke: the Framingham Study. Stroke 2004; 35: 1264-68.

51. Rosenberg G. Inflammation and white matter damage in vascular cognitive impairment. Stroke 2009; 40:S20.

52. Rost N, Wolf P, Kase C, et al. Plasma concentrations of C-reactive protein and risk of ischemic stroke and transient ischemic attack: the Framingham Study. Stroke 2001; 32:257579.

53. Kuo H, Yen C, Chang C, Kuo C Kuo J, Sorond F. Relation of C-reactive protein to stroke, cognitive disorders, and depression in the general population: systematic review and metaanalysis. Lancet Neurol 2005;4:371-80.

54. Elkind M. Inflammatory mechanisms of stroke. Stroke 2010; 41(1): S3S8.

55. Wright $\mathrm{C}$, Moon Y, Paik M et al. Inflammatory markers of vascular risk as correlates of leukoariosis. Stroke 2009; 40:3466-3471.

56. Gorelick P. Role of inflammation in cognitive impairment: results of observational epidemiological studies and clinical trials. Ann N Y Acad Sci 1207 (2010): 155-162.

57. Kalaria R. Cellular, molecular and neuropathological findings in poststroke dementia. J Neurobiol Sci 2009; 283:274.

58. Gorelick P, MD, Bowler J. Advances in Vascular Cognitive Impairment 2007. Stroke 2008;39:279282.

59. Schneider J, Boyle Z, Arvanitakis Z, Bienias J, Bennett D. Subcortical infarcts, Alzheimer's disease, pathology and memory function in older persons. Ann Neurol 2007; 62:59-66.

60. Di Napoli M, Papa F, Bocola V. C-reactive protein in ischemic stroke. Stroke 2001; 32: 917.

61. Komulainen P, Lakka T, Kivipelto $\mathrm{M}$ et al. Serum high sensitivity Creactive protein and cognitive function in elderly women. Age Ageing 2007; 36(4):443-8.

62. Duong T, Acton P, Johnson R. The in vitro neuronal toxicity of pentraxins associated with Alzheimer's disease brain lesions. Brain Res 1998; 813:303-12.

63. Xu G, Zhou Z, Zhu W, Fan X, Liu $\mathrm{X}$. Plasma C-reactive protein is related to cognitive deterioration and dementia in patients with mild cognitive impairment. J of Neurol Sci 2009;284: 7780.

64. Wersching $\mathrm{H}$, Duning $\mathrm{T}$, Lohmann H et al. Serum C-reactive protein is linked to cerebral microstructural integrity and cognitive function. Neurology 2010; 74:1022-1029.

65. Paul A, Ko K, Li L et al. Creactive protein accelerates the progression of atherosclerosis in apolipoprotein E-deficient mice. Circulation 2004; 109: 647-55.

66. Ross R. Atherosclerosis: an inflammatory disease. 1999; 340:115-26.

67. Fornage M, Chiang Y, Meara E,
Psaty B, Reiner A, Sisicovick D. Biomarkers of inflammation and MRI defined small vessel disease of the brain: the cardiovascular health study. Stroke 2008; 39:1952-1959.

68. Hoshi T, Kitagawa K, Yamagami H, Furokado S, Hougaku H, Hori M. Relations of high sensitivity C-reactive protein and interleukin-6 levels with silent brain infarction. Stroke 2005; 36:768-772.

69. Engelhart M, Geerlings M, Meijer J et al. Inflammatory proteins in plasma and the risk of dementia: the Rotterdam study. Arch Neurol 2004; 61:668-72.

70. Ravaglia P, Forti P, Maioli F, Brunetti N, Martelli M. Serum C-reactive protein and cognitive function in healthy elderly Italian community dwellers. J Gerontol A Biol Sci Med Sci 2005; 60(8):1017-1021.

71. Tilvis R, Kahonen-Vare $M$, Jolkkonen J, Valvanne J, Pitkala K, Strandberg T. Predictors of cognitive decline and mortality in aged people over a 10 -year period. J Gerontol A Biol Sci Med Sci 2004; 59:268-74.

72. Schmidt R, Schmidt H, Curb J, Masaki K, White L, Launer L. Early inflammation and dementia: a 25 -year follow-up of the Honolulu-Asia Ageing Study. Ann Neurol 2002; 52: 168-74.

73. Yaffe K, Lindquist K, Penninx B, et al. Inflammatory markers and cognition in well-functioning AfricanAmerican and white elders. Neurology 2003; 61:76-80.

74. Teunissen C, van Boxtel M, Bosma $\mathrm{H}$, et al. Inflammation markers in relation to cognition in a healthy aging population. J Neuroimmunol 2003; 134:142-50.

\author{
Address for correspondence: \\ Maya Danovska, MD, \\ Neurology Clinic, University Hospital Pleven, Bulgaria \\ 8A, George Kochev Str., 5800 Pleven, Bulgaria \\ Tel: +359 64886276 , \\ E-mail: mdanovska@yahoo.com;
}

